\title{
Nuclear dissipation effects on fission and evaporation in systems of intermediate fissility
}

\author{
P. N. Nadotchy ${ }^{1, \text { a }}$, A. Brondi ${ }^{2}$, A. Di Nitto ${ }^{2}$, G. La Rana ${ }^{2}$, R. Moro ${ }^{2}$, E. Vardaci ${ }^{2}$, A. Ordine ${ }^{2}$, A. Boiano $^{2}$, M. Cinausero $^{3}$, \\ G. Prete ${ }^{3}$, V. Rizzi ${ }^{3}$, N. Gelli ${ }^{4}$, F. Lucarelli ${ }^{4}$ \\ 1 Istituto Nazionale di Fisica Nucleare, Via Cinthia, 80126 Napoli, Italy \\ 2 Istituto Nazionale di Fisica Nucleare and Dipartimento di Scienze Fisiche, Universitá di Napoli "Federico II", Via Cinthia, \\ 80126 Napoli, Italy \\ 3 Laboratori Nazionali di Legnaro dell' Istituto Nazionale di Fisica Nucleare, Legnaro (Padova), Italy \\ 4 Istituto Nazionale di Fisica Nucleare and Dipartimento di Fisica, Firenze, Italy
}

\begin{abstract}
The systems of intermediate fissility ${ }^{132} \mathrm{Ce}$ and ${ }^{158} \mathrm{Er}$ have been studied experimentally and theoretically in order to investigate the dissipation properties of nuclear matter. Cross sections of fusion-fission and evaporation residues channels together with charged particles multiplicities in both channels, their spectra, angular correlations and mass-energy distribution of fission fragments have been measured. Theoretical analysis has been performed using multi-dimensional stochastic approach with realistic treatment of particle evaporation. The results of analysis show that full one-body or unusually strong two-body dissipation allows to reproduce experimental data. No temperature dependent dissipation was needed.
\end{abstract}

\section{Introduction}

The dissipation properties of nuclear matter are still an attractive subject in experimental and theoretical investigations with heavy-ion reactions. The nuclear friction accompanying fission process reduces the fission rate and causes its delay to reach a stationary value. During last decades many efforts have been undertaken to a precise determination of the fission time scale, the nature of dissipation and its dependence on the temperature and deformation. At issue is whether nuclear dissipation proceeds primarily by means of individual two-body collision (two-body friction [1]), as the case of ordinary fluids, or by means of nucleons colliding with a moving potential wall (onebody friction [2]). The estimations given by different authors predict a quite wide range of dissipation strengths and different dependencies on temperature and deformation (see reviews $[3,4]$ and references therein). The lack of experimental constraints to the model appears to be, in several cases, one of the source of controversial results.

There are no much experimental data for the systems of intermediate fissility ( $X=0.5-0.6)$ compared to the heavier ones although they offer several advantages. They are characterized by an evaporation residue (ER) cross section comparable or larger than the fusion-fission (FF) cross section. Consequently, the predictions of the theoretical models can be constrained not only by the observables of fusion-fission channel, as it is in the case of heavy nuclei, but also by the observables of the ER channel. The systems of intermediate fissility, compared to the heavier ones, have

\footnotetext{
a e-mail: nadtochy@na.infn.it
}

also larger charged particle multiplicities, which can be investigated together with neutrons. Therefore, the measurements of the relevant quantities in both channels would allow to put severe constraints on the models predictions.

In the present paper we report the results of the theoretical calculations for the reactions $200 \mathrm{MeV}{ }^{32} \mathrm{~S}+{ }^{100}$ $\mathrm{Mo} \rightarrow{ }^{132} \mathrm{Ce}$ and $180 \mathrm{MeV}^{32} \mathrm{~S}+{ }^{126} \mathrm{Te} \rightarrow{ }^{158} \mathrm{Er}$. The comparison with the experimental data for a large number of observables allows us to draw several conclusions about nuclear dissipation.

\section{Experimental setup}

The experiment was performed at the Tandem-ALPI complex of the Laboratori Nazionali di Legnaro (LNL). We used the $8 \pi \mathrm{LP}$ apparatus [5] to detect light charged particles in coincidence with evaporation residues and fission fragments. The fission fragments were detected in the telescopes of the two most forward rings of the BALL section. Evaporation residues have been detected by means of four Parallel Plate Avalanche Counter modules. In a separate experiment ER cross sections are measured by means of the electrostatic deflector of LNL [6] and fission cross sections are measured with the double-arm time-of-flight spectrometer CORSET [7] at LNL as well. We have measured most of the relevant quantities in the ER and FF channels: proton and $\alpha$-particle energy spectra and multiplicities, ER and FF cross sections, and parameters of the fission fragment mass-energy distribution.

This is an Open Access article distributed under the terms of the Creative Commons Attribution-Noncommercial License, which permits unrestricted use, distribution, and reproduction in any noncommercial medium, provided the original work is properly cited. 


\section{Model}

We used a stochastic approach [4] to model fission process dynamically. The evolution of the compound nucleus shape is modelled as the motion of a Brownian particle. The coordinates of this particle (collective coordinates) describe the shape of the compound nucleus and their evolution has been considered using Langevin equations. In the dynamical calculations we used a $\{c, h, \alpha\}$ parametrization [8]. The shape parameters $c, h$, and $\alpha$ have been used to generate the collective coordinates.

The coupled Langevin equations have the form:

$$
\begin{aligned}
\frac{d q_{i}}{d t} & =\mu_{i j} p_{j}, \\
\frac{d p_{i}}{d t} & =-\frac{1}{2} p_{j} p_{k} \frac{\partial \mu_{j k}}{\partial q_{i}}-\frac{\partial F}{\partial q_{i}}-\gamma_{i j} \mu_{j k} p_{k}+\theta_{i j} \xi_{j}(t),
\end{aligned}
$$

where $\mathbf{q}$ is the vector of collective coordinates, $\mathbf{p}$ is the vector of conjugate momenta, $F(\mathbf{q})=V(\mathbf{q})-a T^{2}$ is the Helmholtz free energy, $V(\mathbf{q})$ is the potential energy, $m_{i j}(\mathbf{q})$ $\left(\left\|\mu_{i j}\right\|=\left\|m_{i j}\right\|^{-1}\right)$ is the tensor of inertia, $\gamma_{i j}(\mathbf{q})$ is the friction tensor. The normalized random variable $\xi_{j}(t)$ is assumed to be a white noise. The strength of the random force $\theta_{i j}$ is given by $\sum \theta_{i k} \theta_{k j}=T \gamma_{i j}$. The temperature $T$ has been determined by the Fermi-gas model formula $T=$ $\left(E_{\text {int }} / a\right)^{1 / 2}$, where $E_{\text {int }}$ is the internal excitation energy of the nucleus, and $a$ is the level-density parameter. The repeated indices in the equations above imply summation over the collective coordinates.

The inertia tensor was calculated by means of the WernerWheeler approximation for incompressible irrotational flow [1]. The potential energy of the nucleus was calculated within the framework of a macroscopic model with finite range of the nuclear forces [9]. In the present analysis we have used one-body dissipation [2] based on the "wall" and "wall-plus-window" formulas with a varied reduction factor $k_{s}$ from "wall" formula, and two-body dissipation [1] with the varied two-body friction constant $v_{0}$. Evaporation of prescission light particles $(j=n, p, \alpha)$ along Langevin trajectories was taken into account using a Monte Carlo simulation technique [4]. The partial decay widths of particle emission have been calculated using the statistical code Lilita_N97 [10], which allows to use in the calculations different prescriptions of level density parameter, curvatures of emission barriers, transmission coefficients derived from optical model [11-13] or fusion systematics [14]. We introduced a deformation dependent emission barriers for description of charged particles emission. Thus, we present in this paper preliminary results for calculations with a deformation dependent emission barriers and with emission barriers taken for a spherical shape independent on deformation of compound nucleus.

\section{Results and discussions}

\subsection{Particle multiplicities, FF and ER cross sections}

The cross sections of FF and ER channels together with the mean multiplicities provide quite strong constraints on the input parameters of the dynamical model. The measured characteristics for the compound nuclei ${ }^{132} \mathrm{Ce}$ and ${ }^{158} \mathrm{Er}$ are presented in Table 1 together with the results of dynamical calculations for different values of viscosity coefficient and level density parameter.

In the present calculations the statistical model parameters have been varied in order to fit the multiplicities in ER channel, as they are not to a much sensitive to dissipation strength. In order to get the best fit of observables in FF channel the dissipation strength has been varied. In Table 1 we present the results of calculations, which provide the best description of experimental data for the case of one-body and two-body dissipations.

The calculated results for ${ }^{132} \mathrm{Ce}$ show that the values of $\alpha_{E R}$ will be overestimated changing $a$ from $\mathrm{A} / 6$ to $\mathrm{A} / 8$. Thus, for the best description of experimental data in the case of one-body dissipation one needs to use $k_{s}=1$ and $a=\mathrm{A} / 6$. At the same time the value of $p_{E R}$ will be overestimated approximately by $15 \%$ and $\alpha_{p r e}$ underestimated by $10 \%$ for calculations with spherical emission barriers. In calculations with deformation dependent emission barriers only protons are overestimated by about $2 \%$ in ER and FF channels. In the case of two-body dissipation one needs to use unusually strong value of $v_{0} \simeq 0.15 \times 10^{-21} \mathrm{MeV}$ $\mathrm{s} \mathrm{fm}^{-3}$ in order to get the best description of the experimental data in agreement with previous findings of Ref. [15], where the value $v_{0}=0.125 \times 10^{-21} \mathrm{MeV} \mathrm{s} \mathrm{fm} m^{-3}$ has been found more suitable for the description of experimental $\mathrm{n}_{\text {pre }}, \sigma_{F F}$, and $\sigma_{E R}$ values for ${ }^{200} \mathrm{~Pb}$. Lower values of the dissipation strength (one-body or two-body) will overestimate $\sigma_{F F}$ in our calculations.

The spectra of protons and $\alpha$-particles in ER and FF channels for ${ }^{132} \mathrm{Ce}$ have been also analysed. The calculations with $a=A / 6$ and emission barriers for a spherical shape reproduce well spectra of $\mathrm{p}_{E R}, \alpha_{E R}$, and $\alpha_{p r e}$. However, the $\mathrm{p}_{\text {pre }}$ experimental spectra have a larger temperature. The angular correlations of $\mathrm{p}_{E R}$ and $\alpha_{E R}$ in coincidence with ER are well reproduced in our calculations.

We have obtained similar qualitative behaviour of calculated results by changing the dissipation strength and $a$ for ${ }^{158} \mathrm{Er}$ as for ${ }^{132} \mathrm{Ce}$. Therefore, in Table 1 we present for ${ }^{158} \mathrm{Er}$ only the best results of calculations performed with full one-body dissipation and $a=\mathrm{A} / 9$. We can reproduce prescission particle multiplicities, cross sections of ER and FF channels for ${ }^{158} \mathrm{Er}$, but we can not reproduce $\mathrm{p}_{E R}$ and $\alpha_{E R}$ varying the parameters of the statistical model. This result shows that it is important to measure and analyse observables from both ER and FF channels.

\subsection{Fission timescale}

The time characteristics of the fission process are important for understanding the fission dynamics. One can better estimate the influence of different effects at different stages of fission process. The calculated fission time distribution for ${ }^{132} \mathrm{Ce}$ is presented in Fig. 1 for the case of one-body dissipation with $k_{s}=1$ and $a=A / 6$. The fission time distribution has one maximum at $t_{f}^{\max }=30 \mathrm{zs}$. A fission delay 
Table 1. The experimental and calculated particle multiplicities in the ER and FF channels together with their cross sections.

\begin{tabular}{|c|c|c|c|c|c|c|c|c|c|c|c|}
\hline \multirow[t]{2}{*}{ C.N. } & \multirow{2}{*}{$\begin{array}{l}\text { Emission } \\
\text { bariers }\end{array}$} & \multirow[t]{2}{*}{ Viscosity } & \multicolumn{5}{|c|}{ ER channel } & \multicolumn{2}{|c|}{ FF channel } & \multirow[b]{2}{*}{$\sigma_{\mathrm{FF}}(\mathrm{mb})$} & \multirow[b]{2}{*}{$\sigma_{\mathrm{ER}}(\mathrm{mb})$} \\
\hline & & & $a$ & $n_{E R}$ & $p_{E R}$ & $\alpha_{E R}$ & $n_{\text {pre }}$ & $p_{\text {pre }}$ & $\alpha_{\text {pre }}$ & & \\
\hline \multirow[t]{5}{*}{${ }^{132} \mathrm{Ce}$} & spherical & $k_{s}=1.0$ & $\mathrm{~A} / 6$ & 5.30 & 1.198 & 0.56 & 0.63 & 0.052 & 0.030 & 143 & 793 \\
\hline & spherical & $k_{s}=1.0$ & $\mathrm{~A} / 8$ & 4.98 & 1.20 & 0.70 & 0.80 & 0.075 & 0.061 & 134 & 802 \\
\hline & spherical & $v_{0}=0.15$ & $\mathrm{~A} / 6$ & 5.26 & 1.18 & 0.57 & 0.61 & 0.048 & 0.028 & 125 & 811 \\
\hline & spherical & $v_{0}=0.15$ & $\mathrm{~A} / 8$ & 4.93 & 1.17 & 0.71 & 0.59 & 0.063 & 0.043 & 114 & 822 \\
\hline & deformed & $k_{s}=1.0$ & $\mathrm{~A} / 6$ & 5.37 & 1.07 & 0.56 & 0.80 & 0.063 & 0.033 & 117 & 783 \\
\hline Exp. & & & & & $0.90(14)$ & $0.56(9)$ & & $0.055(7)$ & $0.038(5)$ & $130(13)$ & $828(50)$ \\
\hline${ }^{158} \mathrm{Er}$ & spherical & $k_{s}=1.0$ & $\mathrm{~A} / 9$ & 5.29 & 0.26 & 0.34 & 1.77 & 0.032 & 0.021 & 186 & 789 \\
\hline Exp. & & & & & $0.375(33)$ & $0.234(8)$ & & 0.034 & 0.020 & $195(20)$ & 780 \\
\hline
\end{tabular}
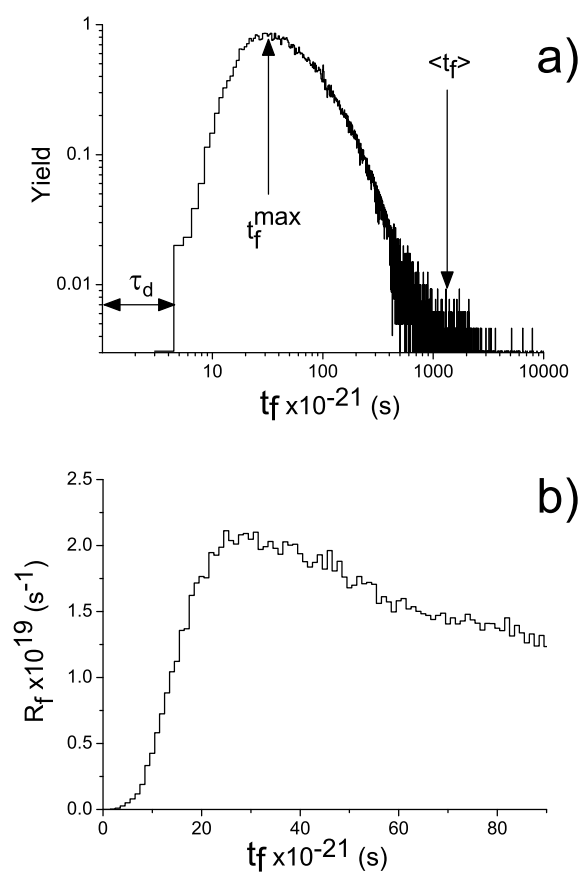

Fig. 1. The fission time distribution (a) and fission rate (b) for ${ }^{132} \mathrm{Ce}$.

time $\tau_{d}=5 \mathrm{zs}$ is found for ${ }^{132} \mathrm{Ce}$, which reflects the dissipation properties of nuclear matter. One can see from this figure that at the time interval $0<t<\tau_{d}$ there is no fission events at all. In the time interval $\tau_{d}<t<t_{f}^{\max }$ the fission time distribution has steep rise from 0 to maximum value. At $t>t_{f}^{\max }$ the fission time distribution is approximately an exponential decreasing function with a long lasting tail up to $10^{-17} \mathrm{~s}$. The arrow at $\mathrm{t}=1250 \mathrm{zs}$ indicates the mean fission time $\left\langle t_{f}\right\rangle$. The fission rate $R_{f}(t)$ has a direct correlation with the fission time distribution. The delay time $\tau_{d}$ corresponds to the $R_{f}(t)=0$. The steep rise of fission time distribution corresponds to the increase of $R_{f}(t)$ till the maximum value at $t_{f}^{\max }$. At the time interval $t>t_{f}^{\max }$ the smooth decrease of $R_{f}(t)$ corresponds to the approximately exponential decrease of the fission time distribution. The $R_{f}(t)$ does not reach a stationary value due to the evaporation of

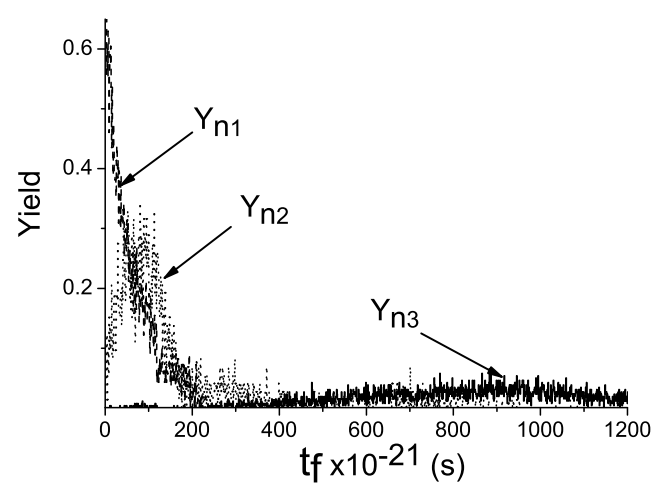

Fig. 2. The yields of first (dashed line), second (dotted line), and third (solid line) prescission neutrons for ${ }^{132} \mathrm{Ce}$.

particles accompanying fission process. For the ${ }^{158} \mathrm{Er}$ compound nucleus we have found $\tau_{d}=9 \mathrm{zs}, t_{f}^{\max }=50 \mathrm{zs}$, and mean fission time is $850 \mathrm{zs}$.

In order to illustrate the time characteristics of the evaporation process we show in Fig. 2 the percentual yields of first $\left(Y_{n 1}(t)\right)$, second $\left(Y_{n 2}(t)\right)$, and third $\left(Y_{n 3}(t)\right)$ prescission neutrons as a function of time. The yields for first prescission proton and $\alpha$-particle are very close to the $Y_{n 1}(t)$. One can see from this figure that $Y_{n 1}(t)$ starts from $\mathrm{t}=0$ and follows to the exponential decrease from maximum at $\mathrm{t}=0$ to 0 at $\mathrm{t} \simeq 250 \mathrm{zs}$. Due to reduction of excitation energy after each evaporation step the emission of every next neutron requires a longer time. The maxima for $Y_{n 2}(t)$ and $Y_{n 3}(t)$ are at $100 \mathrm{zs}$ and $900 \mathrm{zs}$, respectively.

\subsection{The mass-energy distribution of fission fragments}

The mean kinetic energy $\left\langle E_{K}\right\rangle$ and variances of mass $\sigma_{M}$ and kinetic energy $\sigma_{E_{K}}$ distributions for ${ }^{132} \mathrm{Ce}$ are presented in Table 2. For comparison with experimental data we have chosen the calculated results, which provide the best agreement with experimental data for the multiplicities in FF and ER channels.

The mass and kinetic energy distributions of fission fragments are presented in Fig. 3. One can see that the theoretical calculations reproduce quite well the experimental 
Table 2. The experimental and calculated $\sigma_{M}, \sigma_{E_{K}}$ and $\left\langle E_{K}\right\rangle$ values for ${ }^{132} \mathrm{Ce}$ compound nucleus. The calculations have been performed using spherical emission barriers. The experimental value of $\left\langle E_{K}\right\rangle=85.9 \mathrm{MeV}$ has been estimated from Viola's systematics [16]

\begin{tabular}{llllll}
\hline C.N. & Viscosity & $a$ & $\begin{array}{l}\sigma_{M} \\
\text { a.m. u. }\end{array}$ & $\begin{array}{l}\sigma_{E_{K}} \\
\mathrm{MeV}\end{array}$ & $\begin{array}{l}\left\langle E_{K}\right\rangle \\
\mathrm{MeV}\end{array}$ \\
\hline${ }^{132} \mathrm{Ce}$ & $k_{s}=1.0$ & $\mathrm{~A} / 6$ & 14.9 & 7.3 & 82.0 \\
& $k_{s}=1.0$ & $\mathrm{~A} / 8$ & 16.4 & 8.4 & 80.9 \\
& $v_{0}=0.15$ & $\mathrm{~A} / 6$ & 14.6 & 6.7 & 79.6 \\
& $v_{0}=0.15$ & $\mathrm{~A} / 8$ & 16.1 & 8.1 & 78.6 \\
Exp. & & & 15.4 & 11.4 & $90.9(85.9)$ \\
\hline
\end{tabular}

mass distribution. In order to improve the description of experimental mass distribution one could use lower values of $k_{s}$. In this case the variance of mass distribution would become larger or, in other words, the calculated mass distribution would become wider. The kinetic energy distribution of fission fragments can not be reproduced in calculations. The width of experimental kinetic energy distribution is much larger than the calculated one. Similar result has been obtained in 3D Langevin calculations made in Refs. $[17,18]$ and in two-dimensional calculations [15]. In order to reproduce better the experimental values of $\sigma_{E_{K}}$ in dynamical calculations one needs to use lower values of viscosity coefficient $k_{s} \simeq 0.1-0.25$ [18], which does not allow to reproduce the prescission particle multiplicities. Another possible way to improve the theoretical description of the experimental data on $\sigma_{E_{K}}$ could be the use of another parametrization of compound nucleus shape, which could provide more flexible shapes of compound nucleus during the descend from saddle to scission and, as a result, more variability of $E_{K}$. The mean kinetic energy $\left\langle E_{K}\right\rangle$ is better reproduced in the calculations with one-body dissipation than in the calculations with the two-body. The $\left\langle E_{K}\right\rangle$ values obtained in the calculations with two-body viscosity is lower than the experimental one. This result is in agreement with the previous findings [15]. However, we can not rely on the comparison between theoretical and experimental $\left\langle E_{K}\right\rangle$ values, as we can not reproduce well the shape of kinetic energy distribution. Therefore, we can not exclude the large two-body dissipation from analysis of $\left\langle E_{K}\right\rangle$ in the same way as it is done in Ref. [15].

\section{Conclusions}

To summarize the findings of our study, one can conclude that full one-body or unusually strong two-body dissipation allows to reproduce a wide set of observables. The same result has been obtained in Ref. [15], where a limited number of observables have been analysed for fission of ${ }^{200} \mathrm{~Pb}$. The experimental data in FF and ER channels could be reproduced with temperature independent dissipation. The fission delay time of 5 and 9 zs has been obtained for ${ }^{132} \mathrm{Ce}$ and ${ }^{158} \mathrm{Er}$, respectively. The experimental data from ER channel have been used as additional constraints on the models predictions.
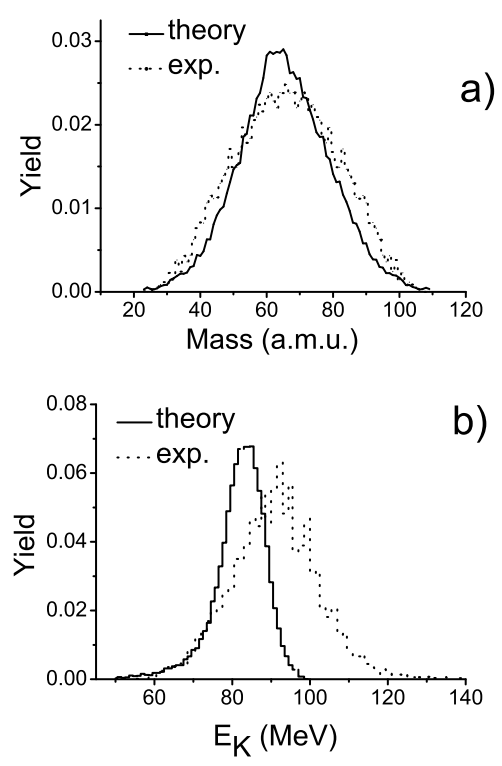

Fig. 3. The experimental and theoretical mass (a) and kinetic energy (b) distributions of fission fragments for ${ }^{132} \mathrm{Ce}$.

\section{References}

1. K. T. R. Davies, A. J. Sierk, and J. R. Nix, Phys. Rev. C 13, (1976) 2385.

2. J. Blocki et al., Ann. Phys. (N.Y.) 113, (1978) 330.

3. D. Hilscher and H. Rossner, Ann. Phys. (Fr.) 17, (1992) 471.

4. P. Fröbrich and I. I. Gontchar, Phys. Rep. 292, (1998) 131.

5. E. Fioretto et al., IEEE Trans. Nucl. Scie. 44, (1997) 1017.

6. S. Beghini et al., Nucl. Instrum. Methods A 239, (1985) 585.

7. E. M. Kozulin et al., Instrum. Exp. Tech. 51, (2008) 44.

8. M. Brack et al., Rev. Mod. Phys. 44, (1972) 320.

9. A. J. Sierk, Phys. Rev. C 33, (1986) 2039.

10. Lilita_N97 is an extensively modified version of the original Lilita program made by J. Gomez del Campo and R. G. Stockstad, Oak Ridge National Laboratory Report No. TM7295, (1981) unpublished.

11. J. R. Huizenga and G. Igo, Nucl. Phys. 29, 462 (1961).

12. F. G. Perey, Phys. Rev. 131, 745 (1963).

13. D. Willmore and P. E. Hudson, Nucl. Phys. 55, 673 (1964).

14. L. C. Vaz et al., Z. Phys. A318, 231 (1984).

15. T. Wada, Y. Abe, and N. Carjan, Phys. Rev. Lett. 70, (1993) 3538.

16. V. E. Viola, K. Kwiatkowski, and M. Walker, Phys. Rev. C 31, (1985) 1550.

17. P. N. Nadtochy, G. D. Adeev, and A. V. Karpov, Phys. Rev. C 65, (2002) 064615.

18. G. D. Adeev et al., Fiz. Elem. Chastits At. Yadra 36, (2005) 732. 